\title{
Electromagnetic wave propagation in time-dependent media with antisymmetric magnetoelectric coupling
}

\author{
Shi-Rong Lin ${ }^{\mathrm{a}}$, Ruo-Yang Zhang ${ }^{\mathrm{b}}$, Yi-Rong Ma ${ }^{\mathrm{a}}$, Wei Jia ${ }^{\mathrm{a}}$, Qing Zhao ${ }^{\mathrm{a}, *}$ \\ ${ }^{a}$ School of Physics, Beijing Institute of Technology, Beijing 100081, P.R. China \\ ${ }^{b}$ Theoretical Physics Division, Chern Institute of Mathematics, Nankai University, Tianjin 300071, P.R. China
}

\begin{abstract}
This paper deals with electromagnetic wave propagation in time-dependent media with an antisymmetric magnetoelectric coupling and an isotropic time-dependent permittivity. We identify a new mechanism of linear birefringence, originated from the combined action of the time-dependent permittivity and the antisymmetric magnetoelectric coupling. Permittivity with linear and exponential temporal variations exemplifies the creation and control of these two distinct types of linear birefringent modes. As a novel nonlinear optical effect, a scheme utilizing optical Kerr effect in moving media is proposed for the realization of the predicted birefringence.
\end{abstract}

Keywords: Time-dependent media, Magnetoelectric effect, Linear birefringence, Moving media, Toroidal moment

\section{Introduction}

Wave propagation in time-dependent media is an intriguing topic. Once introducing time-varying parameters, dynamical properties of macroscopic Maxwell equations dramatically change [1, 2], which deepens our understanding of interactions between light and matter. Indeed, it has been shown that the existence of time-varying parameters of the media can give rise to extraordinary effects such as spectral shifts of radiation [2], velocity modulation of electromagnetic waves [3], time refraction and reflection at the time discontinuous interface [4], frequency-periodic band structure and wave-vector forbidden band gaps in temporal photonic crystal [5], and various particular quantum effects in time-dependent media [6-9]. More specially, it has been demonstrated that the time-dependence of bi-isotropic magnetoelectric coupling will lead to time circular birefringence and time Faraday effect [10].

The previous work on time-dependent media mainly focused on the linear isotropic or bi-isotropic cases. Media with time-dependent and anisotropic parameters, however, have not been fully accounted for. Here, we investigate electromagnetic waves in a particular type of time-dependent magnetoelectric media, which is described by an isotropic time-dependent permittivity, a constant isotropic permeability, and a nonreciprocal antisymmetric magnetoelectric coupling. The nonreciprocal antisymmetric magnetoelectric coupling can be achieved in the moving media $[11,12]$, or through the toroidal moment in toroidalized materials $[11,13]$, such as the polar ferrimagnetic phase of $\mathrm{Ga}_{2-x} \mathrm{Fe}_{x} \mathrm{O}_{3}[14,15]$, the spin-flop phase of antiferromagnetic $\mathrm{Cr}_{2} \mathrm{O}_{3}$ [16], and the magnetic field-induced weakly ferromagnetic phase of antiferromagnetic-incommensurate and

${ }^{*}$ Corresponding author

Email address: qzhaoyuping@bit.edu.cn (Qing Zhao ) ferroelectric $\mathrm{BiFeO}_{3}$ [17]. On the other hand, it may be fabricated in artificial materials including nonreciprocal magnetoelectric coupling response. Such artificial materials have been investigated extensively theoretically [19-24]. Besides, as a practical realization, static and periodically loaded transmission lines have been recognized to emulate moving media [25]. By solving macroscopic Maxwell equations in this time-dependent system, we find some remarkable properties, especially a novel linear birefringent effect, caused by the combined action of the time-dependent permittivity and the magnetoelectric coupling term.

In the following section, we begin with our proposed constitutive relations and obtain the explicit equations governing the dynamics of the electromagnetic waves in such time-dependent media. In Sec 3, we briefly review the dispersion relation in the corresponding time-independent media and find that there is no birefringence in this case despite the anisotropic toroidal coupling. In Sec 4, we proceed to time-dependent case and discover an extraordinary linear birefringent phenomenon. Sec 5 is devoted to the simulations of these birefringent effects. Two specific cases exemplify the creation and control of these two distinct types of linearly polarized modes. In Sec 6, we show that this type of time-dependent magnetoelectric media can be realized through moving media and optical Kerr effect.

\section{Electrodynamics of time-dependent media}

The constitutive relations hide all physics of media in macroscopic electromagnetics. Here, we consider one kind of magnetoelectric constitutive relations

$$
\mathbf{D}=\epsilon(t) \mathbf{E}+\hat{\alpha} \mathbf{H}, \quad \mathbf{B}=\mu \mathbf{H}+\hat{\alpha}^{T} \mathbf{E},
$$


where $\epsilon(t)$ and $\mu$ are time-dependent permittivity and magnetic permeability respectively, and

$$
\hat{\alpha}=\mathbf{g} \times \hat{I}=\left(\begin{array}{ccc}
0 & -g_{3} & g_{2} \\
g_{3} & 0 & -g_{1} \\
-g_{2} & g_{1} & 0
\end{array}\right) .
$$

The parameter $\mathbf{g}$ is the toroidal moment and $\hat{\alpha}$ denotes the antisymmetric magnetoelectric coupling tensor with $\hat{\alpha}^{T}$ representing its transpose. All the parameters are required to be real such that the medium is nondissipative. In writing the constitutive relations (1), we have assumed instantaneous response for the frequency range of interest, and hence, the medium is nondispersive. Noteworthily, the time dependence of $\epsilon(t)$ indicates the effective macroscopic property of the media changing with time. If $\epsilon$ is time-independent, Eqs. (1) may be conceived as effective constitutive relations of moving media. Since $\hat{\alpha}=-\hat{\alpha}^{T}$, according to Lorentz reciprocity theorem, this kind of medium is nonreciprocal [26].

The electrodynamics of the time-dependent media starts from macroscopic Maxwell equations [27]:

$$
\nabla \times \mathbf{E}=-\frac{\partial \mathbf{B}}{\partial t}, \quad \nabla \times \mathbf{H}=\frac{\partial \mathbf{D}}{\partial t}, \quad \nabla \cdot \mathbf{D}=0, \quad \nabla \cdot \mathbf{B}=0 .
$$

We assume no free charge and free current inside the media.

For convenience, we restructure the constitutive relations into

$$
\mathbf{E}=\hat{\varepsilon} \mathbf{D}+\hat{\chi} \mathbf{B}, \quad \mathbf{H}=\hat{v} \mathbf{B}+\hat{\chi}^{T} \mathbf{D}
$$

where $\hat{\varepsilon}=\left(\epsilon-\frac{1}{\mu} \hat{\alpha} \hat{\alpha}^{T}\right)^{-1}, \quad \hat{v}=\left(\mu-\frac{1}{\epsilon} \hat{\alpha}^{T} \hat{\alpha}\right)^{-1}, \quad \hat{\chi}=-\frac{1}{\mu} \hat{\varepsilon} \hat{\alpha}$, and $\hat{\chi}^{T}=-\frac{1}{\epsilon} \hat{v} \hat{\alpha}^{T}$, with superscript -1 representing inverse of a matrix. By substituting Eq. (3) into the first two equations of (2), we have

$$
\nabla \times(\hat{\varepsilon} \mathbf{D}+\hat{\chi} \mathbf{B})=-\frac{\partial \mathbf{B}}{\partial t},
$$

and

$$
\nabla \times\left(\hat{v} \mathbf{B}+\hat{\chi}^{T} \mathbf{D}\right)=\frac{\partial \mathbf{D}}{\partial t} .
$$

For the time-dependent media, we can seek for a class of solutions $\mathbf{D}(\mathbf{r}, t)=\mathbf{D}(t) e^{i \mathbf{k} \cdot \mathbf{r}}$ with a constant wave vector $\mathbf{k}$. We deal with the problem in Cartesian coordinates and set $\mathbf{k}=k \hat{\mathbf{z}}$. With the ansatz $\mathbf{D}(t, k)=\mathbf{D}(t) e^{i k z}$, Maxwell equations imply that $D_{z}=0$ and $B_{z}=0$. Therefore, the Eqs. (4) and (5) transform to

$$
\begin{aligned}
& \hat{\mathbf{x}}: \quad i k\left(\epsilon \mu-g_{2}^{2}\right) D_{y}-i k g_{1} g_{2} D_{x}-i k \epsilon g_{3} B_{x}= \\
& \epsilon\left(\epsilon \mu-g^{2}\right) B_{x}^{\prime}, \\
& \hat{\mathbf{y}}: \quad-i k\left(\epsilon \mu-g_{1}^{2}\right) D_{x}+i k g_{1} g_{2} D_{y}-i k \epsilon g_{3} B_{y}= \\
& \epsilon\left(\epsilon \mu-g^{2}\right) B_{y}^{\prime},
\end{aligned}
$$

and

$$
\begin{aligned}
& \hat{\mathbf{x}}: \quad i k\left(\epsilon \mu-g_{2}^{2}\right) B_{y}-i k g_{1} g_{2} B_{x}+i k \mu g_{3} D_{x}= \\
& -\mu\left(\epsilon \mu-g^{2}\right) D_{x}^{\prime},
\end{aligned}
$$

$$
\begin{aligned}
& \hat{\mathbf{y}}: \quad-i k\left(\epsilon \mu-g_{1}^{2}\right) B_{x}+i k g_{1} g_{2} B_{y}+i k \mu g_{3} D_{y}= \\
& -\mu\left(\epsilon \mu-g^{2}\right) D_{y}^{\prime} .
\end{aligned}
$$

Here, $g^{2}=g_{1}^{2}+g_{2}^{2}+g_{3}^{2}$, and the primed letters denote time derivatives. The Eqs. (6)-(9) are coupled linear ordinary differential equations of $B_{x}(t), B_{y}(t), D_{x}(t)$ and $D_{y}(t)$ with variable coefficients. Obviously, if $\hat{\alpha}=0$, i.e., $g_{1}=0, g_{2}=0$ and $g_{3}=0$, then these equations will reduce to the cases of trivial isotropic media. For time-dependent magnetoelectric media, however, new effects will occur.

\section{Time-independent case}

Before discussing the time-dependent magnetoelectric media, let us first consider the time-independent case as a comparison:

$$
\mathbf{D}=\epsilon \mathbf{E}+\hat{\alpha} \mathbf{H}, \quad \mathbf{B}=\mu \mathbf{H}+\hat{\alpha}^{T} \mathbf{E} .
$$

For such media, the plane wave solutions of the type $e^{i \mathbf{k} \cdot \mathbf{r}-\omega t}$ are admissible, then the Eqs. (4) and (5) reduce to

$$
i \mathbf{k} \times(\hat{\varepsilon} \mathbf{D}+\hat{\chi} \mathbf{B})=i \omega \mathbf{B}, \quad i \mathbf{k} \times\left(\hat{v} \mathbf{B}+\hat{\chi}^{T} \mathbf{D}\right)=-i \omega \mathbf{D}
$$

It is convenient to introduce the following antisymmetric matrix

$$
\hat{k} \equiv \mathbf{k} \times \hat{I}=\left(\begin{array}{ccc}
0 & -k_{z} & k_{y} \\
k_{z} & 0 & -k_{x} \\
-k_{y} & k_{x} & 0
\end{array}\right) .
$$

Hence, we have

$$
(\omega \hat{I}-\hat{k} \hat{\chi}) \mathbf{B}=\hat{k} \hat{\varepsilon} \mathbf{D}, \quad\left(-\omega \hat{I}-\hat{k} \hat{\chi}^{T}\right) \mathbf{D}=\hat{k} \hat{v} \mathbf{B} .
$$

Immediately, eigen equations for $\mathbf{D}$ can be written as

$$
\left[\left(-\omega \hat{I}-\hat{k} \hat{\chi}^{T}\right)-\hat{k} \hat{v}(\omega \hat{I}-\hat{k} \hat{\chi})^{-1} \hat{k} \hat{\varepsilon}\right] \mathbf{D}=0 .
$$

The nontrivial solutions require the following characteristic equation:

$$
\left|\left(-\omega \hat{I}-\hat{k} \hat{\chi}^{T}\right)-\hat{k} \hat{v}(\omega \hat{I}-\hat{k} \hat{\chi})^{-1} \hat{k} \hat{\varepsilon}\right|=0 .
$$

The solutions to the equations given above are

$$
k_{ \pm}=-\omega\left(g_{3} \pm \sqrt{\epsilon \mu-g_{1}^{2}-g_{2}^{2}}\right) .
$$

For a physical conclusion, only positive values of the solutions are acceptable. Hence, provided that $g^{2}<\epsilon \mu$, there will be only one eigenmode for the electromagnetic wave propagating in the time-independent magnetoelectric media.

\section{Time-dependent case}

Let's consider now the time-dependent case. From Eq. (15), it is easily recognized that $g_{1}$ and $g_{2}$ play the same role in the wave propagation when the wave vector is parallel to $z$ direction. Consequently, we can always choose an appropriate coordinate frame such that $g_{2}=0$, then Eqs. (6)-(9) yield

$$
\hat{\mathbf{x}}: \quad i k \mu D_{y}-i k \epsilon g_{3} B_{x}=\left(\epsilon \mu-g_{1}^{2}-g_{3}^{2}\right) B_{x}^{\prime},
$$




$$
\hat{\mathbf{y}}: \quad-i k\left(\epsilon \mu-g_{1}^{2}\right) D_{x}-i k \epsilon g_{3} B_{y}=\epsilon\left(\epsilon \mu-g_{1}^{2}-g_{3}^{2}\right) B_{y}^{\prime},
$$

and

$$
\begin{aligned}
& \hat{\mathbf{x}}: \quad-i k \epsilon B_{y}-i k \mu g_{3} D_{x}=\left(\epsilon \mu-g_{1}^{2}-g_{3}^{2}\right) D_{x}^{\prime}, \\
& \hat{\mathbf{y}}: \quad i k\left(\epsilon \mu-g_{1}^{2}\right) B_{x}-i k \mu g_{3} D_{y}=\mu\left(\epsilon \mu-g_{1}^{2}-g_{3}^{2}\right) D_{y}^{\prime} .
\end{aligned}
$$

Writing $B_{x}$ and $B_{y}$ in terms of $D_{y}$ and $D_{x}$ from Eqs. (18) and (19) respectively, and substituting them into Eqs. (16) and (17), we formulate the evolution equations of the temporal parts of $\mathbf{D}$ as

$$
\begin{aligned}
& \left(\epsilon \mu-g_{1}^{2}-g_{3}^{2}\right) D_{y}^{\prime \prime}+\left(\mu \epsilon^{\prime}+2 i k g_{3}-\frac{\mu \epsilon^{\prime}\left(\epsilon \mu-g_{1}^{2}-g_{3}^{2}\right)}{\epsilon \mu-g_{1}^{2}}\right) D_{y}^{\prime} \\
& +\left(k^{2}+\frac{i k \mu g_{3} \epsilon^{\prime}}{\epsilon \mu-g_{1}^{2}}\right) D_{y}=0,
\end{aligned}
$$

and

$$
\begin{aligned}
& \left(\epsilon \mu-g_{1}^{2}-g_{3}^{2}\right) D_{x}^{\prime \prime}+\left(\mu \epsilon^{\prime}+2 i k g_{3}-\frac{\epsilon^{\prime}}{\epsilon}\left(\epsilon \mu-g_{1}^{2}-g_{3}^{2}\right)\right) D_{x}^{\prime} \\
& +\left(k^{2}-\frac{i k g_{3} \epsilon^{\prime}}{\epsilon}\right) D_{x}=0 .
\end{aligned}
$$

The results reveal a novel linear birefringent effect, since two linearly polarized components of $\mathbf{D}$ evolve independently. If either $\epsilon^{\prime}=0$ or the toroidal moment $\mathbf{g}=0$, these two evolution equations reduce to an identical one. In conclusion, this novel linear birefringent effect is exactly induced by the coupling between the time-varying $\epsilon(t)$ and the toroidal moment $\mathbf{g}$. If the waves propagate along the direction of the toroidal moment, these eigenmodes will evolve in the same way. Consequently, the linear birefringence vanishes in this particular direction which serves as the optical axis of the system.

It is well known that crystalline solids, uniaxial or biaxial materials, result in optical linear birefringence caused by the underlying symmetry of the crystal structure [28]. Similar phenomena occurs in liquids subjecting to external electric field or magnetic field, such as Kerr effect [29], Cotton-Mouton effect [30], magnetoelectric Jones birefringence [31], and magnetoelectric linear birefringence [32]. The linear birefringence discussed here, however, originates from the toroidal moment as well as the time inhomogeneity of $\epsilon$, but not merely from the anisotropy of the material.

\section{Numerical simulations of birefringent modes}

In the preceding section, we identify a new kind of linear birefringence due to the coexistence of time-varying permittivity and $g_{1}$ component of the magnetoelectric coupling tensor. In this section, we will focus on this interesting birefringent phenomenon by investigating two examples. We just consider the condition that the wave propagates in the direction perpendicular to the toroidal moment, i.e., only $g_{1} \neq 0$, however, $g_{2}=g_{3}=0$.
First, let's assume that $\epsilon(t)=\epsilon_{0}(1+\alpha t)$, where $\alpha$ is a constant with the dimension of the inverse of time. In this case, the evolution equations turn out to be

$$
D_{y}^{\prime \prime}+\frac{k^{2} c^{2}}{1+\alpha t-c^{2} g_{1}^{2}} D_{y}=0
$$

and

$$
D_{x}^{\prime \prime}+\left(\frac{\alpha}{1+\alpha t-c^{2} g_{1}^{2}}-\frac{\alpha}{1+\alpha t}\right) D_{x}^{\prime}+\frac{k^{2} c^{2}}{1+\alpha t-c^{2} g_{1}^{2}} D_{x}=0 .
$$

It's obvious that $c^{2}$ is equal to $1 /\left(\epsilon_{0} \mu_{0}\right)$ where $c$ represents the velocity of light. Further, we assume that $\mu=\mu_{0}$. The general analytical solutions to the Eq. (22) are given in terms of modified Bessel functions of the first order $I_{1}$ and $K_{1}$ [33]

$$
\begin{aligned}
D_{y}(t)= & \frac{c k}{\alpha} \sqrt{1+\alpha t-c^{2} g_{1}^{2}}\left[C_{1} I_{1}\left(\frac{2 i c k}{\alpha} \sqrt{1+\alpha t-c^{2} g_{1}^{2}}\right)\right. \\
& \left.+C_{2} K_{1}\left(\frac{2 i c k}{\alpha} \sqrt{1+\alpha t-c^{2} g_{1}^{2}}\right)\right]
\end{aligned}
$$

where $C_{1}$ and $C_{2}$ are constants to be determined by initial conditions. For the Eq. (23), we have to find to numerical solutions. Fig. 1 illustrates the evolution of temporal parts of the two linearly polarized modes $D_{x}$ and $D_{y}$, scaled by the constant electric displacement $D_{x 0}$ and $D_{y 0}$ respectively, in the corresponding time-dependent media. Both the period (frequency) and amplitude of these two modes vary with time. The changing frequency can be roughly explained by the relation $\omega \sim k / \sqrt{\epsilon(t) \mu-g_{1}^{2}}$. Therefore, the frequency decreases as $\epsilon$ increases with time. Further, the amplitude of $D_{y}(t)$ increases with time as the permittivity increases, since the $\sqrt{t}$ dependence of the solution given in the Eq. (24) corresponds to this mode. The mode $D_{x}(t)$ as clearly shown in Fig. 1(a), has similar behavior. Nevertheless, the time-varying amplitude $D_{y}(t)$ experiences a faster increase than that of $D_{x}(t)$. Note that the magnitude of $g_{1}$ also modulates these two modes. By varying the value of $g_{1}$, the magnitudes of the amplitudes change correspondingly (Fig. 1(a) and Fig. 1(b)). Evidently $D_{y}(t)$ decreases with the decrease in $g_{1}$, however, $D_{x}(t)$ increases with the decrease in $g_{1}$. As a consequence, it is impossible to distinguish these two modes as $g_{1}$ tends to 0 (Fig. 1(c)).

Next, consider the exponential type of time-dependent permittivity $\epsilon(t)=\epsilon_{0} e^{\beta t}$, where $\beta$ is a constant with the dimension of the inverse of time. Then, the evolution equations become

$$
D_{y}^{\prime \prime}+\frac{k^{2} c^{2}}{e^{\beta t}-c^{2} g_{1}^{2}} D_{y}=0
$$

and

$$
D_{x}^{\prime \prime}+\frac{\beta c^{2} g_{1}^{2}}{e^{\beta t}-c^{2} g_{1}^{2}} D_{x}^{\prime}+\frac{k^{2} c^{2}}{e^{\beta t}-c^{2} g_{1}^{2}} D_{x}=0
$$

In this case, we numerically solve these two equations given above. Due to the coexistence of the time-dependent permittivity and the toroidal moment, these birefringent modes evolve 

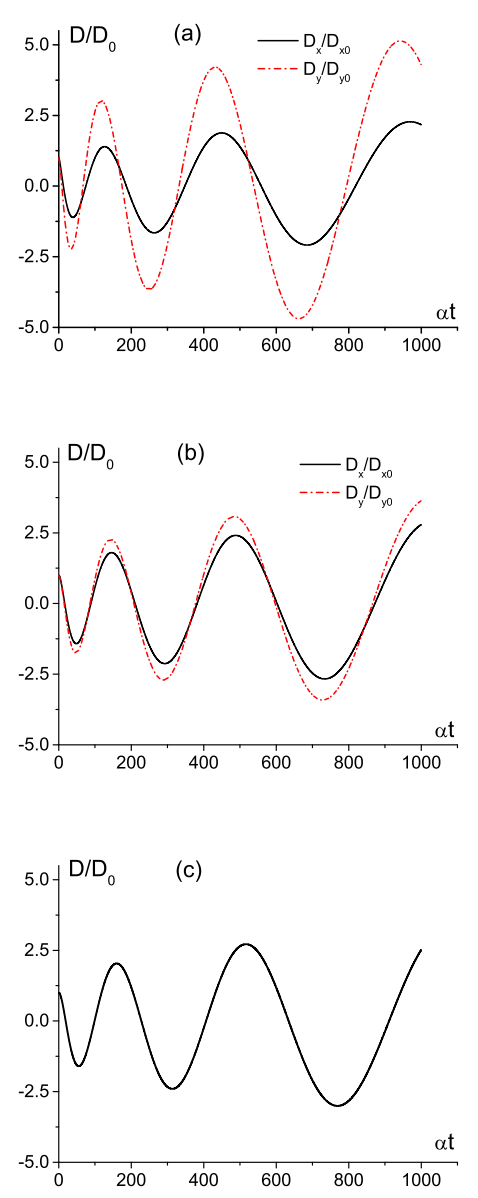

Figure 1: Temporal variations of two linear birefringent modes of the electric displacement modulated by the time-varying permittivity $\epsilon(t)=\epsilon_{0}(1+\alpha t)$. The scaled electric displacement $D_{x}(t) / D_{x 0}$ and $D_{y}(t) / D_{y 0}$ are plotted with solid and dashed curves versus the dimensionless time $\alpha t$, respectively. The initial conditions are set as $D_{x}(0) / D_{x 0}=D_{y}(0) / D_{y 0}=1$, and $D_{x}^{\prime}(0) / D_{x 0}=D_{y}^{\prime}(0) / D_{y 0}=0$. Parameter is chosen as $k=0.1 \alpha / c$. (a) $g_{1}=0.93 / c$, (b) $g_{1}=0.33 / c$, and (c) $g_{1}=0$.

independently as illustrated in Fig. 2(a) and Fig. 2(b). As in the linear time-dependent case, there are similar dynamical wave behaviors. For the two modes, the frequency decreases with time, however, the amplitudes increase with time. Specifically, the amplitude of $D_{y}(t)$ mode manifests a more remarkable changes than that of $D_{x}(t)$ mode. The general trends of $D_{y}(t)$ and $D_{x}(t)$ regarding the change in $g_{1}$ are displayed in Fig. 2(b) and Fig. 2(c), which qualitatively resemble the first example.

We thus know from the above two examples that both linear and exponential increase in the time-dependent permittivity significantly modulate the two linear birefringent modes. Both of which propagate in these kinds of time-dependent media with increasing temporal amplitude as time evolves. The magnetoelectric coupling component $g_{1}$, however, has different effec$\mathrm{t}$ on these modes. Consequently, the linear birefringence can be created and controlled by the combined action of the timedependent permittivity and the magnetoelectric coupling component.
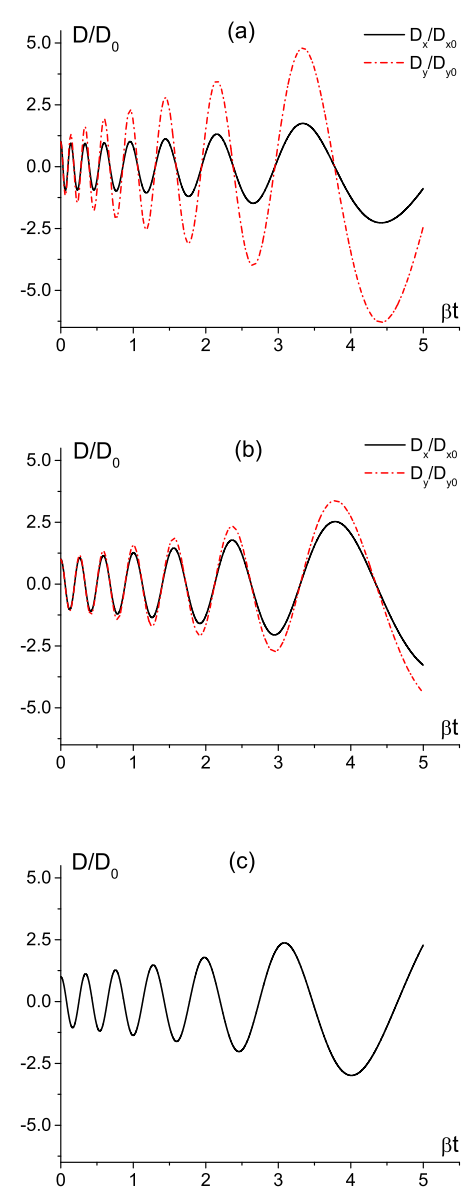

Figure 2: Temporal variations of two linear birefringent modes of the electric displacement modulated by the time-varying permittivity with $\epsilon(t)=\epsilon_{0} e^{\beta t}$. The scaled electric displacement $D_{x}(t) / D_{x 0}$ and $D_{y}(t) / D_{y 0}$ are plotted with solid and dashed curves versus the dimensionless time $\beta t$, respectively. The initial conditions are set as $D_{x}(0) / D_{x 0}=D_{y}(0) / D_{y 0}=1$, and $D_{x}^{\prime}(0) / D_{x 0}=D_{y}^{\prime}(0) / D_{y 0}=0$. Parameter is chosen as $k=20 \beta / c$. (a) $g_{1}=0.93 / c$, (b) $g_{1}=0.67 / c$, and (c) $g_{1}=0$.

\section{Experiment design}

We give an appropriate physical picture of how such kind of time-dependent media is generated. Imagine an isotropic nonlinear medium that uniformly moves at a speed $v$ along $\hat{y}$ direction. An intense pulse polarized in $\hat{z}$ direction passes through the moving media parallel to its moving direction. The pulse will produce a nonlinear polarization that contributes to the variation in permittivity. Consequently, another probe wave travelling into the media along $\hat{z}$ direction will experience this changing refractive index. The situation is displayed schematically in Fig. 3. In the nondispersive and lossless media, the polarization is expressed as a power series in the field strength $E$ as $[34,35]$

$$
P_{i}=\epsilon_{0}\left(\sum_{j} \chi_{i j}^{(1)} E_{j}+\sum_{j k} \chi_{i j k}^{(2)} E_{j} E_{k}+\sum_{j k l} \chi_{i j k l}^{(3)} E_{j} E_{k} E_{l}\right),
$$

where $\chi_{i j}^{(1)}, \chi_{i j k}^{(2)}$ and $\chi_{i j k l}^{(3)}$ denote the linear susceptibility, secondand third-order nonlinear susceptibilities, respectively, and al- 
1 the indexes refer to $x, y$ and $z$. The lowest-order nonlinear contribution to the polarization in the moving media considered here, as isotropic materials, results from the third-order on symmetry grounds. For the present case, we ignore nonlinear contribution higher than third-order.

As a concrete illustration, let the strong wave be a Gaussian pulse $\mathbf{E}_{0}=A e^{-(y-u t)^{2} / a^{2}} e^{i(k y-\omega t)} \hat{\mathbf{z}}$ with an amplitude $A$ and width $a$. Travelling into the moving media, the group velocity of the pulse is given to the first order in $v$ by $u=c / n+v\left(1-n^{-2}\right)$ [12], where $n$ represents the refractive index of the moving media. According to Eq. (27), such an intense pulse will induce nonlinear response $\mathbf{E}[34,35]$. Since only the third-order nonlinearity, i.e. optical Kerr effect, exists in an isotropic material, the polarization corresponding to the probe field can be expressed as

$$
\mathbf{P}=\epsilon_{0}\left(\chi^{(1)}+\chi^{(3)}\left|\mathbf{E}_{0}\right|^{2}\right) \mathbf{E}=\epsilon_{0} \chi \mathrm{eff}(y, t) \mathbf{E},
$$

where $\chi^{(1)}$ and $\chi^{(3)}$ are the effective isotropic susceptibilities. The effective susceptibility reads

$$
\chi_{\mathrm{eff}}(y, t)=\chi^{(1)}+A^{2} e^{-2(y-u t)^{2} / a^{2}} \chi^{(3)}
$$

if we neglect the nonlinear self-modulation of the controlling pulse and assume that it will move with unchanged profile during the interaction with the probe. Consequently, we obtain the effective permittivity

$$
\epsilon(y, t)=\epsilon_{0}\left(1+\chi_{\mathrm{eff}}(y, t)\right)=\epsilon_{0}\left(\epsilon_{1}+\Delta \epsilon(y, t)\right),
$$

where $\Delta \epsilon(y, t)$ is the spacetime-dependent part, while $\epsilon_{1}$ is the spacetime-independent counterpart. Different physical processes can give rise to this effect. Nonresonant electronic polarization is of great importance in that it is ubiquitous in all dielectric media. Typically, $\chi^{(3)}$ covers from $10^{-17} \mathrm{~m}^{2} / \mathrm{V}^{2}$ to $10^{-22} \mathrm{~m}^{2} / \mathrm{V}^{2}$ for the nonresonant electronic nonlinearities [34, 35].

Subsequently, a probe wave propagates into the moving media along $\hat{z}$ direction. The incident direction, parallel to the induced optic axis, is chosen in such a way that birefringent effects caused by the optical Kerr effect is irrelevant here. In the first order approximation with respect to $v / c$, the constitutive relations of the moving media are [27]

$$
\mathbf{D}=\epsilon \mathbf{E}+\left(\epsilon \mu_{0}-\frac{1}{c^{2}}\right) \mathbf{v} \times \mathbf{H}, \quad \mathbf{B}=\mu_{0} \mathbf{H}-\left(\epsilon \mu_{0}-\frac{1}{c^{2}}\right) \mathbf{v} \times \mathbf{E}
$$

Although any variation of the permittivity will lead to the change in the corresponding toroidal moment of the moving media, we note that such spacetime-dependent fluctuation could be neglected since it is proportional to $\Delta \epsilon(y, t) \mathbf{v}$, which is a high order perturbation.

We require that the width $a$ of the controlling pulse is considerably larger than the transverse width of the probe perpendicular to its propagation direction. Then, the probe could be conceived as propagating along a straight line denoting by $y=$ $y_{0}$. As a result, the probe can only experience the nonlinear interaction with the controlling pulse along $y=y_{0}$. For simplicity, we set $y_{0}=0$ and obtain the effective permittivity experienced by the probe $\epsilon\left(y_{0}, t\right) \equiv \epsilon(t)=\epsilon_{0}\left(1+\chi^{(1)}+A^{2} e^{-t^{2} / \tau^{2}} \chi^{(3)}\right)$, where $\tau$ is defined by $\tau=a^{2} /\left(2 u^{2}\right)$ and it is introduced as a "time constant" for the duration of the pulse. Moreover, the transverse width $a_{t}$ of the pulse should be wide enough $\left(a_{t}>u \tau\right)$ so that the probe cannot "feel" the spatial inhomogeneity of the media along the propagating direction during the interaction with the controlling pulse.

Now we examine how the probe varies with time in this moving medium. We numerically solve the corresponding evolution equations as before. Fig. 4(a) and 4(b) depict two scaled birefringent modes $D_{x}(t) / D_{x 0}$ and $D_{y}(t) / D_{y 0}$ versus the dimensionless time $t / \tau$, respectively. Indeed, they evolve independently as shown in Fig. 4(c), where we present $D_{y}(t) / D_{y 0}-$ $D_{x}(t) / D_{x 0}$, the magnitude of their differences evolving in time. In order to evaluate how the velocity $v$ of the moving media affects the linear birefringence, we modulate $v$ while keeping all other parameters fixed. The evolution of $D_{y}$ mode, for example, reveals that both the amplitude and the frequency of the mode can be controlled by $v$, as illustrated in Fig. 5 .

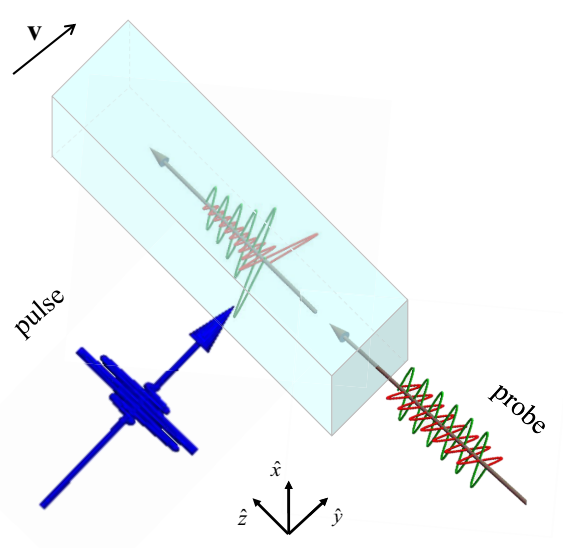

Figure 3: (Color online) Scheme for a realization of time-dependent media via moving media.

\section{Conclusions}

We have investigated electromagnetic wave propagation in a particular type of time-dependent nonreciprocal magnetoelectric medium. In comparison to the time-independent case, we conclude that the coexistence of the time-varying permittivity $\epsilon(t)$ and the toroidal moment $\mathbf{g}$ gives rise to a novel linear birefringence. The direction of the toroidal moment serves as the optical axis of the system. The two eigen polarizations are respectively inside and perpendicular to the plane spanned by the wave vector $\mathbf{k}$ and the toroidal moment $\mathbf{g}$. When the wave propagates along $\mathbf{g}$, the linear birefringence vanishes. In addition, we have visualized this effect through two specific types of time-varying permittivities. Furthermore, we have also proposed an experimental scheme for achieving this striking effect via moving media and optical Kerr effect. This scheme is also the prediction of a novel nonlinear optical effect. Our work provides a new way to control and modulate linearly polarized light. 

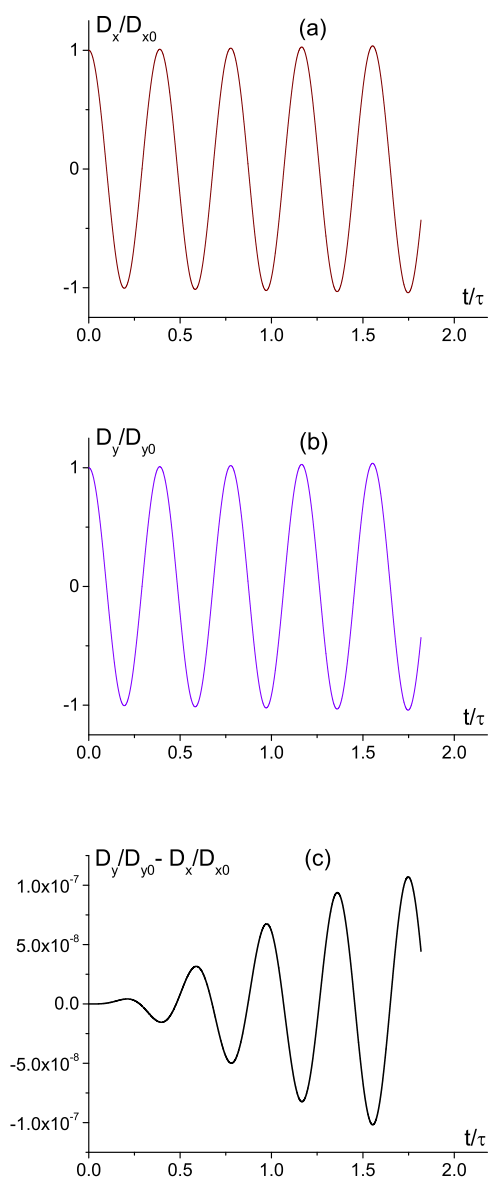

Figure 4: Temporal variations of the scaled birefringent modes of the probe Parameters are chosen as $v / c=0.01, k=55 /(c \tau), \chi^{(1)}=10.56$ and $\chi^{(3)} A^{2}=2.573 \times 10^{-3}$. (a) Evolution of mode $D_{x}(t) / D_{x 0}$; (b) evolution of mode $D_{y}(t) / D_{y 0}$; (c) magnitude of the differences between two scaled birefringent modes evolving in time.

\section{Acknowledgments}

This work is partly supported by the Ministry of Science and Technology of China (2013YQ030595-3, and 2013AA12290 and by NSF of China with the Grant (No. 11275024, and 11475088).

\section{References}

[1] A. B. Shvartsburg, Phys. Usp. 48 (2005) 797.

[2] N.V. Budko, Phys. Rev. A 80 (2009) 053817.

[3] F.R. Morgenthaler, IRE Trans. 6 (1958) 167.

[4] J.T. Mendonca, P.K. Shukla, Phys. Scripta 65 (2002) 160

[5] J.R. Zurita-Sánchez, P. Halevi, J.C. Cervantes-González, Phys. Rev. A 79 (2009) 053821.

[6] J.T. Mendonca, A. Guerreiro, A.M. Martins, Phys. Rev. A 62 (2000) 033805 .

[7] I.A. Pedrosa, A. Rosas, Phys. Rev. Lett. 103 (2009) 010402.

[8] J.R. Choi, Phys. Rev. A 82 (2010) 055803.

[9] J.T. Mendonca, A. Guerreiro, Phys. Rev. A 72 (2005) 063805.

[10] R.-Y. Zhang, Y.-W. Zhai, S.-R. Lin, Q. Zhao, W. Wen, M.-L. Ge, Sci Rep 5 (2015) 13673.

[11] M. Fiebig, J. Phys. D 38 (2005) R123.

[12] U. Leonhardt, T. Philbin, Geometry and Light: The Science of Invisibility, Dover, New York, 2012

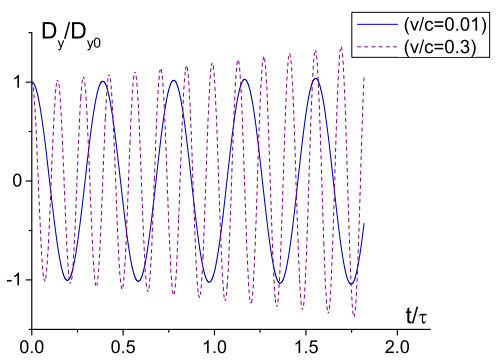

Figure 5: Temporal evolution of the mode $D_{y}(t) / D_{y 0}$ for the cases of $v / c=0.01$ and $v / c=0.3$.

[13] N.A. Spaldin, M. Fiebig, M. Mostovoy, J. Phys. Condens. Matter 20 (2008) 434203

[14] Y.F. Popov, A.M. Kadomtseva, G.P. Vorob'ev, V.A. Timofeeva, M.M. Tehranchi, A.K. Zvezdin, Ferroelectrics 204 (1997) 269.

[15] Y.F. Popov, A.M. Kadomtseva, G.P. Vorob'ev, V.A. Timofeeva, D.M. Ustinin, A.K. Zvezdin, M.M. Tegeranchi, J. Exp. Theor. Phys. 87 (1998) 146.

[16] Y.F. Popov, A.M. Kadomtseva, D.V. Belov, G.P. Vorob'ev, A.K. Zvezdin, JETP Lett. 69 (1998) 330.

[17] Y.F. Popov, A.M. Kadomtseva, A.K. Zvezdin, G.P. Vorob'ev, A.P. Pyatakov, Magnetoelectric Interaction Phenomena in Crystals, edited by M. Fiebig et al., Kluwer, Dordrecht 2004.

[18] A.A. Gorbatsevich, Y.V. Kopaev, Ferroelectrics 161 (1994) 321.

[19] S.A. Tretyakov, A.H. Sihvola, A.A. Sochava, C.R. Simovski, J. Electromagn. Waves. Appl. 12 (1998) 481.

[20] S.A. Tretyakov, Microw. Opt. Technol. Lett. 19 (1998) 365

[21] A. Serdyukov, I. Semchenko, S. Tretyakov, A. Sihvola, Electromagnetics of Bi-anisotropic Materials: Theory and Applications, Gordon and Breach Science, Amsterdam, 2001.

[22] K. Marinov, A.D. Boardman, V.A. Fedotov, N. Zheludev, New J. Phys. 9 (2007) 324.

[23] S.A. Tretyakov, I.S. Nefedov, P. Alitalo, New J. Phys. 10 (2008) 115028.

[24] Y. Ra'di, V.S. Asadchy, S.A. Tretyakov, IEEE Trans. on Antennas Propagation. 61 (2013) 4606.

[25] J. Vehmas, S. Hrabar, S. Tretyakov, New J. Phys. 16 (2014) 093065.

[26] J.A. Kong, Electromagnetic Wave Theory, Wiley-Interscience, New York, 1975.

[27] L.D. Landau, E.M. Lifshitz, L.P. Pitaevskii, Electrodynamics of Continuous Media, Butterworth-Heinemann, Oxford, 1984

[28] M. Born, E. Wolf, Principles of Optics, Cambridge University Press, Cambridge, 1999.

[29] J. Kerr, Philos. Mag. 50 (1875) 337; 50 (1875) 416.

[30] A. Cotton, M. Mouton, Compt. Rendu. 141 (1905) 317; 141 (1905) 349; 145 (1907) 229; 145 (1907) 870.

[31] T. Roth, G.L.J.A. Rikken, Phys. Rev. Lett. 85 (2000) 4478.

[32] T. Roth, G.L.J.A. Tikken, Phys. Rev. Lett. 88 (2002) 063001.

[33] M. Abramovitz, I.A. Stegun, Handbook of Mathematical Functions, Dover, New York 1972

[34] Y.R. Shen, The Principles of Nonlinear Optics, Wiley, New York, 1984.

[35] R.W. Boyd, Nonlinear Optics, Elsevier, Amsterdam, 2008. 